\title{
“El primer usurpador fue el Santo"Cooperativa de Vivienda San Sebastián, un ejemplo de hacer política pública por mano propia ${ }^{1}$.
}

RESUMEN: En el actual contexto caracterizado por la pauperización de amplios sectores sociales, donde existe una gran dependencia de los subsidios y planes distribuidos desde las distintas agencias del Estado, el margen de acción y empoderamiento que tienen estos grupos es realmente muy estrecho. En este marco, la experiencia de autoorganización de un grupo de familias de escasos recursos, que dio por resultado la conformación de la Cooperativa de Vivienda San Sebastián, constituye sin duda una excepción. Sin embargo, esta experiencia abrió la posibilidad de concebir soluciones alternativas a las tradicionales. Además de dar respuesta a sus necesidades básicas más inmediatas esta iniciativa permitió una relación con el Estado, y con distintos sectores de la sociedad civil, desde un lugar distinto al de la pasividad que supone ser objeto de la asistencia pública.

Los habitantes del barrio San Sebastian lograron resolver el grave problema producido por la ausencia de políticas públicas destinadas a garantizar el acceso a la vivienda. Pero, además, esta experiencia interpeló al Estado de forma tal que logró forzar la generación de políticas públicas desde las instancias que tienen la responsabilidad y la obligación de generarlas.

PALABRAS CLAVE : ciudadanía - inclusión/exclusión - políticas públicas - empoderamiento

ABSTRACT: In the present context characterized by the pauperization of ample social sectors, where a great dependency of the subsidies and plans distributed from the different agencies from the State exists, the margin of action and empowerment that have these groups is really very narrow. In this frame, the experience of autoorganization of a group of families of limited resources, that he gave by result the conformation of the Cooperative of House San Sebastian, it constitutes without a doubt an exception. Nevertheless, this experience opened the possibility of conceiving alternative solutions to the traditional ones. Besides to give to answer to its immediate basic necessities this initiative it allowed a relation with the State, and with different sectors from the civil society, from a place different from the one from the passivity that supposes to be object of the public attendance.

\footnotetext{
${ }^{1}$ Ponencia presentada en la “Conference The Social Police and Challenges of Equity and Citizenship” Olinda , Pernambuco, Brazil 08 a 10 de Marzo de 2006.

2 Pesquisadora del Instituto de Desarrollo Económico y Social (IDES) - Instituto de Ciencias Antropológicas, Sección Etnología y Etnografía, Facultad de Filosofía y Letras, Universidad Nacional de Buenos Aires (UBA). Güemes $41734^{\circ} \mathrm{B}$

Ciudad Autónoma de Buenos Aires - Argentina.laurace@argentina.com
} 
The inhabitants of the district San Sebastian managed to solve the serious problem produced by the absence of public policies destined to guarantee the access to the house. But, in addition, this experience interhair to the State so that it managed to force the generation of public policies from the instances that have the responsibility and the obligation to generate them.

KEYWORDS: Citizenship, Inclusion/Exclusion, Public Policies, Empowerment 


\section{El contexto nacional}

La década de los 90’ se caracterizó por el alto nivel de precarización de la vida cotidiana de un amplio sector de la sociedad argentina. Dimensiones fundamentales, tales como el trabajo, la salud, la educación y la vivienda se vieron seriamente afectadas por la implementación de las políticas neoliberales que dominaron la década y que terminaron colapsando y dando como resultado la crisis institucional (además de económica) que se desató a fines del 2001. La situación social del país se refleja en el índice de pobreza creciente, una importante disparidad geográfica en la distribución del ingreso, elevado y creciente nivel de desempleo, y un importante aumento de la informalidad laboral ${ }^{3}$.

Paralelamente el proceso de descentralización impactó en el diseño y distribución de políticas públicas de modo diferencial al interior del país. Algunas regiones y localidades se vieron beneficiadas con esta estrategia, mientras que otras se vieron

perjudicadas. En localidades con un alto grado de movilización social como, por ejemplo, Cutral Co (en la provincia de Neuquén), la descentralización permitió articular desde el Municipio local distintos planes sociales impulsados desde el Estado Nacional y la entidad subestatal provincial. En otros lugares donde la movilización social y el nivel de protesta fue escaso o nulo, o donde la articulación entre los niveles Estatales y subestatales fueron poco fluidos, el impacto efectivo de las políticas públicas fue menor e intermitente.

De todos modos, en términos generales, las políticas públicas aparecieron en este contexto más como una respuesta de urgencia a las demandas sociales que como fruto de una planificación de Estado a mediano y largo plazo.

\footnotetext{
${ }^{3}$ Los datos expuestos fueron relevados del informe elaborado por Acuña, C.; Kessler, G. y Repetto, F. (2002) “Evolucion de la politica social argentina en la decada de los noventa: cambios en su logica, intencionalidad y en el proceso de hacer la politica social”. Proyecto Self-Sustaining Community Development in Comparative Perspective. Coordinado por el Center for Latin American Social Policy CLASPO- The University of Texas at Austin. En el mismo los autores dan cuenta de que el índice de pobreza alcanzó en mayo de 2002 el 50 \%, habiendo partido de un piso de $30 \%$ en 1990; mientras que el ingreso per cápita de la jurisdicción más rica es 4.1 mayor al de la más pobre; a esto se suma un 18,4 \% de desocupación para octubre de 2001 y un 40 \% de trabajadores en relación de dependencia en estado de informalidad laboral.
} 
Se trató de planes destinados principalmente a amortiguar los efectos devastadores de la pobreza creciente, y en menor medida de la exclusión social ${ }^{4}$. Tal como observa Quinti (1997, p74)., a diferencia de la pobreza, la exclusión social es un

“fenómeno producido por la interacción de una pluralidad de procesos (o factores) elementales que afectan a los individuos y a los grupos humanos, impidiéndoles acceder a un nivel de calidad de vida decente, y/o participar plenamente, según sus propias capacidades, en el proceso de desarrollo”

En este sentido, las políticas públicas en la década pasada apuntaron más a satisfacer las necesidades básicas, que a promover procesos de “inclusión social”; como consecuencia de este accionar, las políticas públicas en general no llegaron a contener y disminuir los “factores de riesgo social”, como por ejemplo, las dificultades de acceso a la instrucción, al trabajo, a los servicios sociales, al crédito o a la vivienda.

Los sectores vulnerables se vieron entonces impulsados a buscar las formas de inclusión por sus propios medios, prescindiendo de la asistencia Estatal o, llegado el caso, inclusive confrontando con el Estado a fin de forzar su inserción en el campo de la ciudadanía.

\section{El contexto local}

La ciudad de Cipolletti se encuentra en el Alto Valle de la Provincia de Río Negro, lindante a la ciudad capital de la vecina provincia de Neuquén. La provincia de Río Negro se encuentra en el norte de la Patagonia Argentina, su población se nuclea en las regiones económicamente más activas, los valles de intensa producción agropecuaria y la ciudad de San Carlos de Bariloche, importante centro turístico. Esta provincia presenta grandes espacios despoblados y desigual distribución de la población con una densidad de 2,5 hab./km2. El Índice de Desarrollo Humano es de 0.870 según los cálculos realizados en 1994 y tiene un 20,7 \% de su población con NBI (Necesidades Básicas Insatisfechas). El $28 \%$ de las viviendas de la provincia son deficitarias, en tanto que el hacinamiento alcanza

${ }^{4}$ Los planes y subsidios destinados a la población desocupada fueron los más extendidos. 
al $10 \%$ de las unidades habitacionales. El 82\% de la población cuenta con red pública de provisión de agua y el servicio de cloacas alcanza el $46 \%$ de las viviendas del Alto Valle ${ }^{5}$.

En este marco la ciudad de Cipolletti, con 75.000 habitantes, y una estructura productiva dependiente en gran media de la fruticultura, presenta fuertes déficit en la capacidad de promover, desde la instancia Municipal, el desarrollo y la llegada de los servicios básicos a una amplia franja de la población carenciada.

Uno de los problemas más importantes es la escasez de viviendas que resulten accesibles a este sector de la población que se encuentra desocupado, subocupado o dependiendo de empleos estacionales, propios de la actividad agrícola.

Esto contrasta con la explosión de la construcción de viviendas y el aumento significativo de los costos de las mismas ya sea en relación a los precios de venta como de renta. Sin embargo, la construcción es consecuencia de la inversión privada y se encuentra íntimamente relacionada con el negocio inmobiliario, por lo tanto las unidades habitacionales apuntan a los sectores medio- altos y altos de la sociedad local.

Este proceso que se está dando actualmente en la ciudad de Cipolletti, está en relación directa con el estado del mercado inmobiliario en la vecina ciudad de Neuquén, capital de la provincia del mismo nombre. Neuquén es una ciudad que cuenta con alrededor de 280.000 habitantes, y ha tenido en los últimos años un crecimiento explosivo que no logró ser acompañado por una planificación urbana acorde. Los inmuebles en la ciudad de Neuquén tienen un costo de entre un 50 y un 70 \% mayor a los valores que se manejan en la vecina ciudad de Cipolletti.

Esta gran inversión en obras de construcción de viviendas por parte de los capitales privados, contrasta con la escasa oferta de viviendas sociales y la prácticamente inexistente inversión de los distintos niveles del Estado en la construcción de planes habitacionales. La consecuencia de esta ausencia de política pública destinada a promover el acceso a la vivienda a los sectores más desprotegidos derivó en un fenómeno que se extendió desde mediados de lo '90 en la ciudad de Neuquén y hacia fines de la misma década y principio

${ }^{5}$ Estos datos se encuentran en el Informe del PNUD “En búsqueda de la igualdad de oportunidades”. 2002. Argentina.

CADERNOS GESTÃO PÚBLICA E CIDADANIA, V. 11, N. 49 - JUL/DEZEMBRO 2006 
de la actual comenzó a darse también en la ciudad rionegrina de Cipolletti, nos referimos a la usurpación de tierras fiscales o privadas.

Las “tomas", como se las denomina localmente, son asentamientos extremadamente precarios, resultantes de las profundas desigualdades en el acceso a la propiedad. Estos asentamientos no cuentan con ninguno de los servicios básicos (luz, gas, red cloacal, agua potable). En general el agua y la luz son también "tomadas" a partir de las instalaciones que se encuentren más cercanas al asentamiento, mediante extensiones de construcción casera, por lo que son sumamente precarias y muy inseguras. La calefacción, de vital importancia en esta zona de clima frío, se resuelve principalmente mediante la combustión de leña y, en menor medida, se utilizan artefactos eléctricos.

Los materiales con los que se realizan estas casillas son cajas de cartón desarmadas, nylon (proveniente de bolsas en desuso), listones de madera, chapas de cartón para los techos. Los pisos no cuentan con ningún tipo de aislación, por lo que en los pisos de tierra se suele dejar un espacio para el fogón en el que se quema la leña para calefaccionar o cocinar.

La localización de las “tomas” en ambas ciudades está en directa relación con los predios que se encuentren en estado de abandono. Si bien en general estos predios están ubicados mayormente en las zonas marginales de ambas ciudades, también se instalan “tomas” en terrenos no utilizados que se encuentran en zonas estratégicas y cercanas a los centros de ambas ciudades.

Los procesos que conllevan las “tomas” hasta que logran algún nivel de inclusión, aunque sea de manera desprolija en el ejido urbano, muestran ciertas regularidades, especialmente en la ciudad de Neuquén, donde este fenómeno tiene cierta antigüedad. Una vez instalada la “toma” comienzan una serie de reclamos tanto al Municipio como al Estado provincial con el fin de conseguir la regularización de la tenencia de la tierra y el acceso a materiales de construcción para mejorar las viviendas. Transformar una "toma” en "barrio" es el objetivo principal de los ocupantes de estos asentamientos, ya que se trata de pasar de la ilegalidad a la legalidad. Cuando se consiguen los títulos de propiedad de las tierras que se ocupan, se puede acceder a la regularización de los servicios básicos y se consigue una posición más favorable en la relación de fuerzas con respecto al Municipio. 
El extendido y tradicional sistema clientelar que rige las relaciones políticas y sociales en Neuquén, permite que este pasaje de “toma” a "barrio” sea bastante frecuente. La entrada a la legalidad, del mismo modo que el acceso a los materiales de construcción, se acelera de manera significativa en los tiempos preelectorales.

En la vecina ciudad de Cipolletti esta dinámica no es tan frecuente, ni se encuentra tan aceitada, entre otras cosas, porque se trata de un fenómeno mucho más reciente. En general las pocas "tomas” que se hicieron han entrado rápidamente en procesos judiciales con el objetivo de lograr el desalojo de los intrusos. Aun en este contexto, donde la intervención de las entidades estatales y subestatales es mucho menor, existe la tendencia a demandar al Estado las soluciones a esta problemática, contando con un bajo nivel de empoderamiento y asunción de protagonismo por parte de los afectados directos.

La experiencia de la Cooperativa de Vivienda San Sebastián en la Ciudad de Cipolletti, provincia de Río Negro.

A partir del mes de agosto del año 2003, comenzaron a llegar ocupantes que se instalaron en terrenos privados de la ciudad de Cipolletti ${ }^{6}$, llegando a conformar un grupo de 250 familias. El predio se encontraba abandonado, lo único que había en él era una ermita dedicada a San Sebastián. Al conformarse la toma, sus habitantes se auto identificaron como “toma San Sebastián” dejando a un lado la denominación de “usurpadores de la tierra de Zoppi”, que desde los medios de prensa locales se les había asignado. Los protagonistas recuerdan: “cuando llegamos a las tierras de Zoppi, no había nada, era un páramo. Lo único que había era el San Sebastián. Así que el primer usurpador fue el Santo”.

Las familias fueron llegando lentamente y se fueron conociendo a partir de la convivencia en ese espacio y la necesidad de resolver problemas comunes y urgentes: el acceso a la luz, el acarreo de agua potable de la única canilla a la que tenían acceso y que se encontraba a $400 \mathrm{~m}$ del predio. Se trataba de un grupo de familias muy jóvenes en su mayoría, con niños pequeños y con trabajos precarios y de baja calificación. Los adultos del grupo contaban con las herramientas básicas de la lecto-escritura, algunos tenían el nivel primario

\footnotetext{
${ }^{6}$ Circunscriptos por las calles Juan Domingo Perón, Esquiú, Venezuela y Pastor Bowdrel. Estos terrenos pertenecen a la familia Zoppi
} 
terminado, otros no y excepcionalmente algunas mujeres había llegado a cursar los primeros años de la educación media.

Un grupo importante de las familias que constituyeron esta "toma” vivían previamente en viviendas alquiladas, otros convivían en estado de hacinamiento con las familias paternas. La llegada a la “toma” no fue fácil para este grupo de familias acostumbradas a tener acceso a los servicios básicos. El objetivo desde un principio fue acceder a sus propias casas y mejorar su calidad de vida. Surge así la necesidad de organizarse. Luego de varias reuniones deciden realizar el aporte de una cuota social, con el fin de constituir

un fondo común que les permitiera emprender la compra financiada de las tierras que ocupaban.

Sin embargo, mientras este incipiente proceso de autoorganización se conformaba, tres oficiales de justicia y un abogado llegaron a la "toma” para entregar a los ocupantes una intimación con un plazo de 48 horas para abandonar las tierras y evitar así el inminente desalojo por parte de la fuerza pública.

El propietario del predio había radicado la denuncia con el fin de recuperar sus tierras y no se encontraba dispuesto a generar una instancia de dialogo mientras la usurpación continuara. Las familias involucradas, por su parte, afirmaban que eran conscientes de que su accionar era considerado ilegal, pero a la vez hacían notar la inutilización del terreno y su intención de comprarlo.

Paralelamente, los ocupantes de la toma, comenzaron a reunirse con la autoridades políticas de la ciudad, mientras su nivel de organización iba creciendo. En estas reuniones, los representantes de la “toma” San Sebastián, comentaron su iniciativa de generar un plan de ahorro con el objetivo de comprar el terreno. Sin embargo, desde el Municipio se tomó como decisión política, no apoyar a los habitantes de la toma con el argumento de que "la Municipalidad no sería cómplice de un delito "7.

Los ocupantes empezaron a percibir como una urgencia tan importante como la de acceder a una terreno propio y a una vivienda digna, la necesidad de salir de la ilegalidad, ya que

${ }^{7}$ Declaraciones hechas al Diario local “La mañana de Cipolletti”, 1 de noviembre, 2003. 
desde este lugar de “ocupantes ilegales”, o “usurpadores”, veían afectada seriamente su capacidad de agencia para intervenir o dialogar con las autoridades. Deciden organizarse como cooperativa, sin embargo no contaban con la formación, ni la información necesaria para encarar el proyecto. Una vez más recurren al Municipio local en busca de orientación y apoyo, sin embargo las autoridades vuelven a negarles la asistencia ya que consideran que sus condiciones socioeconómicas no les permiten acceder a un tipo de organización de la complejidad de una cooperativa.

Recurren entonces a las autoridades provinciales y consiguen acceder a un curso sobre cooperativismo que les permite luego conformarse como cooperativa, logrando en el mes de abril de 2005 la inscripción oficial en el Estado nacional como "Cooperativa de Vivienda San Sebastián”.

Sin duda, las tensiones entre el Municipio y el Estado provincial, que responden a diferentes signos políticos, actuó en este caso como un facilitador para la conformación de la cooperativa. Las disputas internas entre los distintos partidos que luchan por la capitalización de los potenciales votantes, abrió una brecha en la que este grupo de familias encontró la posibilidad de darle legitimidad al espacio de construcción de derecho que venían autogestionándose.

En este sentido, algunos antecedentes importantes que permitieron concebir una actuación de conjunto de mayor envergadura, fueron: la comisión de madres y la puesta en funcionamiento de "la escuelita”. La comisión de madres fue formada por cinco mujeres que se organizaron para cocinar diariamente para las familias más necesitadas y preparar la merienda a los 60 niños que vivían en la “toma”. Para recaudar fondos y destinarlos a la compra de alimentos, realizaron rifas con lo cual compraron alimentos básicos como fideos, arroz, azúcar y leche. Además trabajaron en busca de donaciones y lograron que empresas alimenticias locales hicieran un aporte semanal solidario.

Una de las inquietudes de las familias era lograr un apoyo escolar para sus hijos, con esfuerzo comunitario armaron una construcción precaria a la que llamaron "la escuelita”, luego buscaron otros apoyos solidarios, logrando que jóvenes estudiantes de magisterio acompañaran a sus hijos en las tareas escolares.

CADERNOS GESTÃO PÚBLICA E CIDADANIA, V. 11, N. 49 - JUL/DEZEMBRO 2006 
Sin duda, otro elemento que colaboró de manera importante en la consolidación del grupo fue el actuar conjuntamente a la hora de la manifestación pública de sus reclamos. Los cortes de calles frente al Municipio en reiteradas oportunidades, las cartas y notas enviadas a las autoridades locales y a los medios de comunicación, entre otras medidas fueron dándoles un marco de legitimidad a su accionar y una identidad propia al grupo que les permitió ganar terreno en el campo de la construcción de derecho y de una ciudadanía activa que por su calidad de usurpadores les era negada.

Finalmente, el aporte solidario que venían realizando de manera conjunta mensualmente, y la legalidad que les otorgaba el haberse constituido como entidad cooperativa, les permitió presentarse en las inmobiliarias locales como potenciales "clientes" para la compra de terrenos propios.

Luego de recorrer sin éxito varias de las inmobiliarias de la ciudad, lograron que una reconocida empresa local les brindara la oportunidad que necesitaban. Así llegaron a comprar dos amplios lotes donde podrían instalarse ya no como usurpadores, sino como propietarios.

$\mathrm{Al}$ desocupar las tierras del particular se levantó la denuncia que pesaba sobre ellos. En un día de Pascua del año 2005, mudaron sus precarias viviendas a los lotes que habían comprado. Las incomodidades persistían, pero el logro era importantísimo, habían conseguido con ese paso salir de la ilegalidad, y adquirir un terreno del que ya no podrían desalojarlos. Sin embargo, quedaban muchos problemas por resolver ya que para que los servicios básicos llegaran a los lotes adquiridos debían ser reconocidos por el Municipio como "barrio".

Las tensiones entre la ya Cooperativa de Vivienda San Sebastián y las autoridades Municipales se acentuaron. El intendente de Cipolletti le envía al empresario inmobiliario que les había vendido los lotes a la Cooperativa una nota en la cual le exige información sobre la compra-venta realizada con la gente del barrio San Sebastián. El empresario, por su parte, responde enviando una carta documento en la cual reclama al Intendente que se rectifique ya que considera que los términos de la nota dirigida a él son altamente ofensivos 
y declara: "por el planteo que me ha hecho el municipio me da la sensación de que a

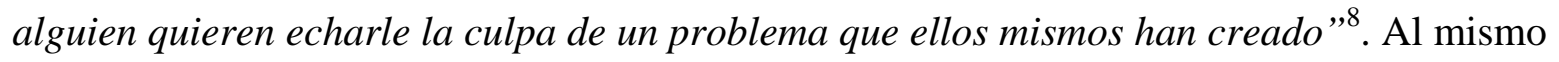
tiempo expresa que no descarta iniciar acciones legales contra las autoridades de la comuna, ya que en la medida que se trata de una operación privada, no tiene porque brindar la información que se le exige desde el

municipio, solo podría solicitársele por vía judicial si hubiera algún proceso de por medio, y este no era el caso.

Paralelamente, el Municipio aplicando las reglamentaciones vigentes para la organización del espacio urbano, exigió a la Cooperativa que ampliara los lotes adjudicados a cada familia y dispusiera al interior de los terrenos adquiridos por ellos parcelas de uso fiscal, destinadas a espacios verdes. Sin el cumplimiento de estas condiciones el Municipio no reconocería a estos asentamientos como nuevos barrios. Los miembros de la Cooperativa respondieron dándole al municipio un plazo de diez días para que les proporcionara un subsidio que les permitiera comprar tierras donde ubicar a las ochenta familias que debían sacar de los predios ya adquiridos para cumplir con el requerimiento del municipio, en el caso contrario, el planteo no sería aceptado.

Mientras las disputas con el Municipio se acentuaban, los miembros de la Cooperativa San Sebastián comenzaron a tener sus primeros contactos con Nación, a través de la Secretaría de Acción Social. El Gobierno Nacional se comprometió a comprar los lotes donde reubicar a las familias y los que eran necesarios para destinar a los espacios verdes. Además, les prometieron su ayuda para la construcción de viviendas de material.

Actualmente, y a pesar de que las gestiones con Nación permitieron cumplir con los requerimientos municipales, la gente de la Cooperativa San Sebastián sigue aguardando a que se los habilite como barrio para así poder solicitar a las distintas entidades proveedoras de servicios, los servicios básicos que necesitan. Sin embargo, a pesar de lo conflictivo que resultó este proceso, la habilitación como "barrio" es inminente, y por otra parte recientemente desde el Municipio se lanzó un programa de apoyo para al conformación de

${ }^{8}$ Diario “La Mañana de Cipolletti”, Martes 5 de Abril de 2005.

CADERNOS GESTÃo PÚBLICA E CIDADANIA, V. 11, N. 49 - JUL/DEZEMBRO 2006 
cooperativas de viviendas sociales, con el fin de controlar el problema de las "tomas” ya que, como el caso de la Cooperativa San Sebastián demostró, la organización y autogestión popular resulta altamente efectiva.

La experiencia positiva y los logros alcanzados por este grupo de familias las induce a potenciar su organización, actualmente están trabajando para conseguir la construcción de forma colectiva de sus casas de material, y simultáneamente consideran ampliar las funciones de la Cooperativa. Efectivamente, su próximo objetivo es conformar una cooperativa de trabajo.

\section{La interpelación de la experiencia}

En un marco de pauperización de amplios sectores sociales, donde existe una gran dependencia de los subsidios y planes distribuidos desde las distintas agencias del Estado, el margen de acción y empoderamiento que tienen estos grupos es realmente muy estrecho. La dinámica predominante es la de la dependencia y el sometimiento a las reglas del juego clientelar.

Las autoridades Municipales de la ciudad de Cipolletti apostaron al desgaste, a la creación de conflictos internos en el grupo, a la “incapacidad” que suponían podían tener estas familias para realizar y sostener algún tipo de organización debido a la escasez de recursos materiales y simbólicos. Partían del supuesto de que la únicas políticas públicas destinada a estos sectores debían ser aquellas dirigidas a cubrir las necesidades mínimas para la subsistencia (las bolsas de comida, la leña), y las destinadas a la "seguridad”, ya que estos sectores en "riesgo social” son considerados potencialmente como proclives al delito.

El diseño de políticas públicas dirigidas a garantizar los derechos y la promoción del desarrollo social de estos sectores no se considera una prioridad en la agenda gubernamental. Sin embargo, la perspectiva de los actores es diferente, en ocasión de uno de los tantos reclamos dirigidos al Intendente local, los miembros de este grupo expresaban lo siguiente: “(...)tenga en cuenta que si bien para usted, somos cabecitas negras o la escoria 
de Cipolletti, como nos han denominado en varias ocasiones, también somos ciudadanos de Cipolletti (...)”9. Es importante destacar la necesidad de reafirmar su cualidad de ciudadanos ante el poder político, aparentemente la situaciones de carencia ocultan la ciudadanía de los sectores sociales vulnerables ante los ojos del poder. Quizás el término más dramático, y al miso tiempo esperanzador, de lo expresado sea "también"; ya que muestra de forma explícita que el grupo tiene conciencia de que se encuentra absolutamente naturalizado, y por lo tanto legitimado, el hecho de que las condiciones de existencia determinan las posibilidades de su inclusión como ciudadanos. Asumiendo esta realidad, la cuestionan, no solo discursivamente sino a partir de una serie de acciones que tienden a asumir, representar y posicionarse en la trama de relaciones de fuerza desde su cualidad de ciudadanos a pesar de la precariedad de sus condiciones materiales.

Por otra parte existe un fuerte desfasaje entre la capacidad de planificación y autogestión de este grupo, y las respuestas lentas, a destiempo y muy poco operativas por parte de las agencias del Estado. Sin bien la confrontación directa fue con el Municipio, el apoyo otorgado tanto desde el Estado provincial como Nacional fue tardío y complicado. Al respecto Ziccardi observa: "los ciudadanos y las organizaciones sociales que demandan aún la atención de sus necesidades urbanas básicas dedican grandes esfuerzos y tiempo para identificar a los interlocutores gubernamentales con capacidad e interés en resolver sus demandas. La complejidad y la ineficiencia burocrática, así como la falta de información respecto a los criterios de asignación de recursos y/o definición de prioridades en las instituciones del aparato gubernamental para tratar asuntos de interés público, son indicadores de la debilidad de las democracias latinoamericanas y de los obstáculos que existen para que la ciudadanía pueda ejercer sus derechos sociales (algunos de los cuales están consagrados en las respectivas constituciones). A esta situación debe atribuirse, en parte, el que durante décadas la forma de relación entre los movimientos urbanos autónomos y las instituciones del Estado fuese de enfrentamiento y lucha.” (2001, 113).

\footnotetext{
${ }^{9}$ Carta dirigida al Intendente de la ciudad de Cipolletti, Alberto Wereltilneck, firmada por la Comisión de Madres, el día 23 de Noviembre del 2004.
} 
El éxito de esta experiencia resonó en otros grupos instalados en “tomas” tanto de la misma ciudad como de la vecina ciudad de Neuquén, donde el problema se encuentra

mucho más extendido. Los miembros de la Cooperativa son convocados asiduamente por los habitantes de otras "tomas” con el fin de explicar cómo consiguieron organizarse autónomamente y resolver el problema.

En efecto, en la generación de una organización independiente de las fuerzas partidarias, de las organizaciones religiosas y de los aparatos gubernamentales se visualiza una manera más efectiva y directa de acceder a los derechos ciudadanos.

Paralelamente, se va generando una red entre incipientes organizaciones que buscan generar sus propios espacios de autonomía y establecen solidaridades que les permite ir construyendo identidades inclusivas. La conformación de pequeñas organizaciones medianamente articuladas y solidarias entre sí potencia significativamente la capacidad de agencia de cada grupo.

Otro aspecto importante es la generación de saberes que se van capitalizando a partir de estas experiencias. Las peripecias atravesadas por este grupo de familias para lograr una organización con reconocimiento oficial fueron innumerables, por otro lado tuvieron que aprender competencias que les resultaban absolutamente desconocidas, como por ejemplo, aquellas relacionadas con los movimientos bancarios. Estos saber también se ponen en circulación y permiten a otros grupos transitar con menos obstáculos por el camino de la gestión y auto organización.

Finalmente, esta experiencia fue tomada por las propias autoridades locales que encontraron en este sistema de conformación de cooperativas una estrategia válida para dar solución al problema del acceso a la vivienda de los sectores desprotegidos.

Los habitantes del barrio San Sebastián lograron sobreponerse y resolver el grave problema producido por la ausencia de políticas públicas destinadas a garantizar el acceso a la vivienda. Pero además esta experiencia interpeló al Estado de forma tal que logró forzar la generación de políticas públicas desde las instancias que tienen la responsabilidad y la obligación de generarlas. Ya que disponen de los recursos necesarios para eso, en la medida 
que es competencia del Estado el garantizar las condiciones que permitan a sus habitantes el ejercicio de una ciudadanía efectiva.

\section{Reflexiones finales}

A la hora del análisis de las lógicas de la política pública y las responsabilidades ciudadanas es importante tener en cuenta la disparidad en la capacidad de agencia de los actores. Las marcadas desigualdades que decantan en una fuerte segmentación y fragmentación social impactan en el nivel de participación ciudadana.

En Argentina, las distintas dimensiones de la ciudadanía se encuentran garantizadas para un porción mínima de la población, mientras que amplios sectores sociales deben luchar por conquistar su cualidad de ciudadanos. Si bien en términos formales la ciudadanía civil que hace referencia a las libertades individuales (igualdad ante la ley, libertad de opinión, de pensamiento, de religión, de propiedad); la ciudadanía política asociada fundamentalmente al sufragio universal y la posibilidad de elegir o ser electo; y la ciudadanía social referida a los derechos a la educación, la salud, la seguridad social, la vivienda, se encuentran garantizados por la carta magna para todos los habitantes del país, en términos reales una porción significativa de la población se encuentra fuera de este marco. Especialmente en relación a la dimensión de la ciudadanía social es donde se encuentran las falencias más importantes, el alto nivel de exclusión pone en duda la operatividad de la democracia, en la medida que tal exclusión implica la negación de los derechos fundamentales. Tal como observan Jelin y Hershberg, la exclusión y la marginalidad son lo opuesto a la idea de actores y escenarios.

Es función del Estado diseñar políticas públicas que tiendan a garantizar, promover y desarrollar el ejercicio de una ciudadanía plena para la mayoría de los ciudadanos, si este piso común no es previsto desde las instancias gubernamentales, el sentido de lo público, del derecho y del mismo sistema democrático se torna difuso, lejano e insignificante para amplios sectores de la sociedad civil. La democracia se reduce al ejercicio eleccionario, momento en que los sectores vulnerables pueden aspirar a ejercer 
una mínima presión obteniendo algún recurso de primera necesidad en la medida que se comporten como clientes leales.

Las instancias Estatales y subestatales, en la media que no dispongan de políticas públicas que den respuesta a los problemas que el propio sistema genera, deben apoyar y acompañar las iniciativas de la sociedad destinadas a generar niveles importantes de autonomía.

Los grupos desprotegidos que fuerzan su inclusión en una ciudadanía real, tomando en sus propias manos la resolución de problemas sociales graves, comprometen al resto de los sectores sociales más acomodados que tienen una capacidad de agencia mayor muchas veces desaprovechada. Efectivamente a nivel local, son pocas las organizaciones civiles constituidas por los sectores medios y altos, destinadas a ejercer un control responsable sobre el accionar del Estado, especialmente en relación a la distribución de los recursos y las prioridades en la agenda pública.

Al mismo tiempo el accionar de estos grupos que sin recursos materiales ni simbólicos, logran articular de manera exitosa la legalidad con la legitimidad implica una fuerte interpelación al poder político que muchas veces se apoya en la legalidad para sostener prácticas y políticas ilegítimas, haciendo un uso poco transparente o mal planificado del recurso público.

Finalmente, resulta urgente mejorar la articulación entre las iniciativas de la sociedad civil y las instancias estatales, a fin de que no se perciba al entramado organizacional de la sociedad como un "problema" a sortear sino como un marco de contención al cual respetar y a partir del cual lograr una convivencia pacífica. Al respecto los integrantes de la Cooperativa expresaban:

"nosotros hicimos todo solos, nadie nos ayudó en nada, más bien nos pusieron un montón de trabas. No queremos al Estado ¿para qué? cuando aparecen es para sacarte plata. Si no saben qué hacer, que no hagan nada, que nos dejen a nosotros, pero que no se metan después a querer hacer cumplir reglas que no sirven para nada".

Si bien la experiencia relevada resultó altamente positiva es importante tener en cuanta que en la medida que se vayan generando instancias de resolución de problemas en 
forma paralela al Estado se van creando esferas o circuitos diferenciados que pueden tender a profundizar la segmentación y la fragmentación social, creando "sociedades" dentro de “sociedades”, cada una con su lógica propia y con un bajo nivel de compatibilidad entre sí.

El desafío es encontrar los caminos para lograr que estas iniciativas y construcciones de ciudadanía con autonomía permitan volver realmente permeable los límites de la sociedad mayor. A fin de que, desde el Estado hasta los distintos sectores sociales, se vean comprometidos en la ampliación y el desarrollo de los mecanismos de inclusión.

\section{Bibliografía}

ACUÑA, C.; Kessler, G. y Repetto, F.) "Evolucion de la politica social argentina en la decada de los noventa: cambios en su logica, intencionalidad y en el proceso de hacer la politica social". Proyecto Self-Sustaining Community Development in Comparative Perspective. Coordinado por el Center for Latin American Social Policy -CLASPO- The University of Texas at Austin, 2002.

Informe del PNUD En búsqueda de la igualdad de oportunidades. Argentina.,2002.

JELIN, E. y HERSHBERG, E. Desarrollos convergentes, diversidad y cambio: imágenes de los derechos humanos. En: Construir la democracia: derechos humanos, ciudadanía y sociedad en América Latina. Venzuela: Nueva Sociedad, 1996.

QUINTI, G. Exclusión social: sobre medición y sobre evaluación. En. MÉNJIVAR, D. y LIETEKE(Coord) Pobreza, exclusión y política social. Costa Rica: FLACSO,1997

ZICCARDI, A. Las ciudades y la cuestión social. En: Pobreza, desigualdad social y ciudadanía. Los límites de las políticas sociales en América Latina. Argentina: CLACSO, 2001.

Artigo recebido em 20/05/2006 e aprovado em 10/10/ 2006. 